\title{
Chronic kidney disease among the Iranian-Azari population; a report from pilot phase of AZAR cohort study
}

\author{
Fariba Mahmoodpoor ${ }^{1}$, Mohammad-Reza Ardalan ${ }^{1 *}$, Mohammadhossein Somi $^{2}$, Elnaz Faramarzi ${ }^{2}$, Sepideh \\ Zununi Vahed ${ }^{1}$, Mousa Ghaoyr Nahand ${ }^{1}$
}

${ }^{1}$ Kidney Research Center, Tabriz University of Medical Sciences, Tabriz, Iran

${ }^{2}$ Liver and Gastrointestinal Diseases Research Center, Tabriz University of Medical Sciences, Tabriz, Iran

\section{A R T I C L E I N F O}

Article Type:

Original

\section{Article History:}

Received: 10 October 2017

Accepted: 2 January 2018

Published online: 19 January 2018

\section{Keywords:}

Renal diseases

Chronic kidney disease

Persian cohort

End-stage renal disease

\begin{abstract}
A B S T R A C T
Introduction: Chronic kidney disease (CKD) is a worldwide health problem. Most patients with CKD are asymptomatic and unaware of their disease until reaches its latter stages. The worldwide prevalence of CKD is increasing and delayed diagnosis takes from the patients the opportunities for early treatment.

Objectives: Our cohort, named AZAR cohort, is a part of a national cohort program and it is aimed to study the major cardiovascular, pulmonary, renal, diabetes, and cancers diseases risk factors in East-Azerbaijan province in North West of Iran.

Patients and Methods: In this cross-sectional study, all eligible subjects (35-65 years) were recruited. Information about demographic, medical history, some physical and para-clinical were collected. Here, we report the results of pilot phase of this study.

Results: Findings showed that prevalence of CKD (stage 3, eGFR $<60 \mathrm{~mL} / \mathrm{min}$ ) among our studied population was $8 \%$ (71/898). The studied population was ranged from young adult to pre-elderly (35-65 years). We found a higher proportion of CKD in women (M/F 14/57). The total proportion of diabetics in our study was 126 persons (14\%). Distribution of different stages of CKD was as follows: stage 3A (67 persons, M/F; 14/53), stage 3B (4 females), stage 2 (737 persons, M/F; 328/409) and stage 1 (88 persons, M/F; 64/24). There were no cases of CKD stage 4 or 5 in our cohort. Body mass index (BMI), serum triglyceride (TG), and cholesterol levels had a significant correlation with CKD stage $3(P<0.05)$. Diabetic patients and female gender were at increased risk of CKD stage 3 (OR: 1.5; 95\% CI: 0.857-2.861).

Conclusion: The prevalence of CKD stage 3 in our cohort was compatible with other previous publications. The higher prevalence of CKD in middle aged to early elderly women population could be explained by the high prevalence of obesity among this population.
\end{abstract}

Implication for health policy/practice/research/medical education:

Early diagnosis and implementation of simple preventive measurements are the key elements to fight against the risk of CKD. In this regard awareness about the prevalence of CKD and its probable risk factors are the first steps toward those goals.

Please cite this paper as: Mahmoodpoor F, Ardalan MR, Somi M, Faramarzi E, Zununi Vahed S, Ghaoyr Nahand M. Chronic kidney disease among the Iranian-Azari population; a report from pilot phase of AZAR cohort study. J Renal Inj Prev. 2018;7(3):124-128. doi: 10.15171/jrip.2018.30.

\section{Introduction}

Chronic kidney disease (CKD) is a worldwide health problem. Most patients with CKD are asymptomatic and unaware of their kidney disease until reaches its later stages (1). The worldwide prevalence of CKD is estimated between $8 \%$ to $16 \%$ and it is increasing. It seems that this upward trend is more prominent among low and middle-income population with limited access to health care. The proportion of end-stage renal disease (ESRD) population who are impending to start the dialysis vastly underestimates global CKD burden because pre-dialysis CKD exceeds ESRD prevalence by as much as 50 times 
$(2,3)$. Sometimes nonspecific symptoms of CKD direct patients toward different specialties. Delayed diagnosis takes from the patients the opportunities for early treatment. CKD not only progress to the ESRD but also is a major risk factor for cardiovascular disease (CVD). Early detection of kidney diseases and timely referral to nephrology improved the patients' outcomes (4). The traditional approach recommends screening among patients with diabetes mellitus, hypertension or CVD. For increasing the screening coverage consideration of older than 60 years population and low socioeconomic status (SES) has been proposed. Early stages of CKD can be detected by simple methods such as urine dipstick and serum creatinine measurement (4-6).

\section{Objectives}

In this study we assessed the prevalence of $\mathrm{CKD}$ in Iranian-Azari population.

\section{Patients and Methods}

\section{Study design and setting}

Azar cohort is a prospective population-based study and a part of a national screening program named Persian cohort. It is aimed to study the major non-communicable diseases risk factors, including cardiovascular, pulmonary, renal, diabetes and cancers disease. Azar cohort is started from October 2014 and still it is continuing in EastAzarbaijan province in North West of Iran. It is expected up to 15000 persons within the age range of 35-70 years will enter the study. Our study was based on the results of pilot phases of the Azar cohort that was gathered between October 2014 up to January 2015.

During the pilot phase all eligible subjects of a small city named 'Khameneh' were studied. In our study we entered the population between the ages of 35-65 years. The exclusion criteria were pregnancy and unwillingness to participate.

\section{Measurements}

In this study all participants underwent general history taking and physical examination. History of presence of diabetes, hypertension, cardiovascular disease, herbal medicine taking and dietary habit were taken. Anthropometric measurements including height, weight, waist, and hip and also wrist circumference were recorded. Blood pressure was measured twice with 10 minutes apart from both upper extremities separately and the mean of measurements was recorded as the blood pressure of each person. From each individual $17 \mathrm{~mL}$ of blood were taken after at least 12 hours of fasting, for following measurements; fasting blood glucose (FBS), hemoglobin $A_{1 C}$, lipids profile, liver enzymes, complete cell blood count (CBC), thyroid hormones (T3,T4,TSH), C reactive protein (CRP), ESR. Additionally serum calcium, phosphorus, sodium, potassium and blood urea nitrogen (BUN) was assessed. Serum creatinine was measured by Jaffe reaction. Urinary protein excretion, glucosuria and urinary ketone were evaluated by the dipstick test (Acon -china LCA5721$01)$. Second screening by urine dipstick was performed in those who had proteinuria and hematuria in their first screening. A 24 hours proteinuria measurement was conducted for those who had proteinuria and hematuria. Individuals with persistent hematuria were referred to nephrology clinics for detection of dysmorphic red blood cells. Those with GFR $<45 \mathrm{~mL} / \mathrm{min}$ were also referred to nephrology clinic.

Estimated glomerular filtration rate (eGFR) was calculated using the simplified Modification of Diet in Renal Disease (MDRD) study equation $(7,8)$. Body mass index (BMI) was also calculated.

Diabetes mellitus was identified as a documented history of diabetes mellitus or elevated blood glucose (FBS $\geq 126$ $\mathrm{mg} / \mathrm{d}$ ).

Aperson's blood pressure was calculated as the average of the two measurements in each arm. Subjects with SBP $\geq 140$ and $\mathrm{DBP} \geq 90$ or self-reported having hypertension were defined as patients with hypertension.

Our definition of CKD was based on the National Kidney Foundation Kidney Disease Outcomes Quality Initiative working group definition of CKD (7).

CKD stages were defined as follows: stage 1, GFR $\geq 90 \mathrm{~mL} /$ $\mathrm{min} / 1.73 \mathrm{~m}^{2}$, stage 2; GFR between $60-89 \mathrm{~mL} / \mathrm{min} / 1.73$ $\mathrm{m}^{2}$; stage 3 , GFR between $30-59 \mathrm{~mL} / \mathrm{min} / 1.73 \mathrm{~m}^{2}$, stage 3a (46-59), 3b (30-45), stage 4; GFR between $15-29 \mathrm{~mL} /$ $\mathrm{min} / 1.73 \mathrm{~m}^{2}$; and stage 5 , GFR $<15 \mathrm{~mL} / \mathrm{min} / 1.73 \mathrm{~m}^{2}$. In our study we only considered persons with CKD stage 3 or higher as our screening target (eGFR below $60 \mathrm{~mL}$ / $\min (7)$.

\section{Ethical issues}

This cross-sectional study was approved by Ethics Committee of Tabriz University of medical sciences (tbzmed.rec.1393.205). The research followed the tenets of the Declaration of Helsinki. Written informed consent was obtained from all patients and remained confidential.

\section{Statistical analysis}

The results of the study are presented as frequencies, percentages, mean \pm standard deviation. We used simple statistical methods for descriptive parameters such as age, weight, high and blood pressure measurements. We used conditional logistic regression analysis for estimating crude and adjusted odds ratios (OR) and their respective 95\% CIs. The correlation of different stages of CKD with hypertension, BMI, cholesterol was evaluated by Spearman's correlation coefficient test. The analysis was conducted using the SPSS software (Statistical Package for the Social Sciences, version 11.5) and $P$ value less than 0.05 was considered significant.

\section{Results}

Baseline characteristics of the studied population are presented in Table 1. Around 898 (M/F: 406/492 with ages of 35 to 65 years) were included in the study. Proportion 
of diabetics in our study was 126 persons (14\%) and 28 of them were newly diagnosed in our cohort. The mean of BMI in males and females with stage $3 \mathrm{CKD}$ was as the following $\left(30.12 \pm 4.32 \mathrm{~kg} / \mathrm{m}^{2}\right.$ vs $26.54 \pm 3.66 \mathrm{~kg} /$ $\left.\mathrm{m}^{2} ; \mathrm{P}=0.004\right)$. Around, 71 persons (\%8 M/F 14/57) had a reduced eGFR (defined as a value $<60 \mathrm{~mL} / \mathrm{min} / 1.73$ $\mathrm{m} 2$ ). Distribution of different stages of CKD were as the following; stage3A (67 persons, M/F; 14/53), stage 3B (4 female), stage $2(737$ persons, $\mathrm{M} / \mathrm{F} ; 328 / 409)$ and stage 1 (88M/F; 64/24) (Table 2). We did not find persons with CKD stage 4 or 5 in our cohort. After one year in second measurements of eGFR only 42 from 71 people who were discovered as CKD stage 3 remained in this group, and 29 persons were returned to CKD stage 2 . All individuals with CKD stage $3 \mathrm{~B}$ or those with persistent proteinuria and hematuria were referred to nephrology clinic for future follow up.

The presence of hypertension tentatively increases the risk of CKD stage 3 two times (OR: 2; 95\% CI: 0.90-5.57). $\mathrm{BMI}$, triglyceride (TG), and cholesterol had a meaningful correlation with CKD stage $3(P<0.05)$. Diabetic patients were at increased risk of CKD stage 3(OR: 1.5 ; 95\% CI: 0.857-2.861).

\section{Discussion}

The result of our study revealed that prevalence of CKD (stage 3, eGFR $<60 \mathrm{~mL} / \mathrm{min}$ ) among our studied population (35-65 years) was $8 \%$. The studied population was ranged from young adults to pre-elderlies who

Table 1. Demographic characteristics of participants $(n=898)$

\begin{tabular}{|c|c|c|c|}
\hline & Male & Female & Total \\
\hline Age (y) & $49.9 \pm 8.026$ & $48 \pm 7.67$ & $48.9 \pm 7.9$ \\
\hline Weight (kg) & $78.5 \pm 13.7$ & $72.6 \pm 13.3$ & $75.2 \pm 13.7$ \\
\hline Height (cm) & $172 \pm 6.8$ & $157.4 \pm 6.2$ & $164 \pm 9.7$ \\
\hline BMI $\left(\mathrm{kg} / \mathrm{m}^{2}\right)$ & $26.5 \pm 4.2$ & $29.3 \pm 5$ & $28 \pm 4.8$ \\
\hline Cholesterol (mg/dL) & $179.89 \pm 37.2$ & $191.21 \pm 38.6$ & $186.1 \pm 38.3$ \\
\hline $\mathrm{HDL}(\mathrm{mg} / \mathrm{dL})$ & $43.9 \pm 9.6$ & $51.4 \pm 10.5$ & $48 \pm 10.8$ \\
\hline Triglyceride (mg/ dL) & $160.7 \pm 88.1$ & $155.6 \pm 83.7$ & $157.9 \pm 86.1$ \\
\hline \multicolumn{4}{|l|}{ Blood pressure } \\
\hline Systolic (mm Hg) & $109 \pm 15$ & $106.5 \pm 15.4$ & $107.6 \pm 15.3$ \\
\hline Diastolic (mm Hg) & $69.6 \pm 8.5$ & $68.3 \pm 8.2$ & $68.9 \pm 8.4$ \\
\hline $\begin{array}{l}\text { Fasting blood } \\
\text { glucose (mg/ dL) }\end{array}$ & $102.4 \pm 32.2$ & $103.9 \pm 37.5$ & $103.2 \pm 35.2$ \\
\hline
\end{tabular}

are making the most active part of society. We did not investigate pediatrics, teenagers and elderlies ( $>65$ years), hence the result of our study could not extrapolate to the general population. We found a higher proportion of CKD in women. In our study BMI had a positive correlation with CKD prevalence. The higher prevalence of CKD in women could be explained by the high prevalence of obesity among this population. Presumably lower levels of recreational physical activity and regular sport activities could account for the high prevalence of obesity and observed difference in CKD prevalence. The positive correlation between high cholesterol and triglyceride levels and CKD prevalence is also connected to this explanation. As we found in our study, hypertension by itself and as a part of metabolic syndrome is associated within increment of CKD. Importantly, we did not discover any patient with advanced CKD (stage 4 or 5 ). Age limit of our study $(<65)$ may be an explanation.

In the first 25 years of life the most common causes of CKD are congenital anomalies of the kidneys and urinary tract, steroid-resistant nephrotic syndrome (SRNS), chronic glomerulonephritis and renal cystic diseases and ciliopathies. Monogenic cause of renal disease can be detected in up to $20 \%$ of those individuals. Because of our age selection ( $>35$ years) we cannot estimate the above conditions in our study. In another CKD screening program that was performed in Golestan province of Iran the prevalence of CKD stage 3-5 was 5\% among the 3591 participants ( $>18$ years) (9). In a study on $>60000$ individuals from Rumania, the prevalence of CKD (eGFR $<60 \mathrm{~mL} / \mathrm{min}$ ), or positive urinary dipstick test was $6.69 \%$. The mean age of the studied population was 55 years and CKD was more common in elders (10). The approximate prevalence of CKD stage 3 among adults over the age of 65 was $11 \%$ in a report from the United States. A selected (>40 years) population study on 6200 individuals in northern Italy showed a prevalence of $6.4 \%$ for CKD stage 3 (11). Likewise, in a study from the Netherlands the reported prevalence of CKD was $5.8 \%$ (mean age was 49 years) (12). The trend of increased prevalence of CKD with increasing the age was preserved in all above studies and also in a recent cross-sectional study from Spain (13). Serum creatinine as a screening biomarker has several limitations and delays identification of the

Table 2. The prevalence of CKD stratified by age and gender

\begin{tabular}{|c|c|c|c|c|c|c|}
\hline \multirow{2}{*}{ CKD stages } & \multicolumn{3}{|c|}{ Female, No. (\%) } & \multicolumn{3}{|c|}{ Male, No. (\%) } \\
\hline & $35-44$ & $45-54$ & $55-65$ & $35-44$ & $45-54$ & $55-65$ \\
\hline Stage 1 (GFR $\geq 90 \mathrm{ml} / \mathrm{min}$ ) & $14(8.3)$ & $8(3.6)$ & $2(2)$ & $26(23.6)$ & $27(15.5)$ & $11(9)$ \\
\hline Stage 2 (GFR=60-89 $\mathrm{ml} / \mathrm{min})$ & $148(88)$ & $183(82)$ & $78(78.8)$ & $83(75.5)$ & $144(82.8)$ & $101(82.8)$ \\
\hline Stage $3 a(G F R=46-59 \mathrm{ml} / \mathrm{min})$ & $6(3.8)$ & 31 (13.9) & $16(16.2)$ & $1(0.9)$ & $3(1.7)$ & $10(8.2)$ \\
\hline Stage $3 \mathrm{~b}(\mathrm{GFR}=30-45 \mathrm{ml} / \mathrm{min})$ & 0 & $1(0.45)$ & $3(3)$ & 0 & 0 & 0 \\
\hline Stage 4 (GFR=15-29 ml/min) & 0 & 0 & 0 & 0 & 0 & 0 \\
\hline Stage 5 (GFR<15 ml/min) & 0 & 0 & 0 & 0 & 0 & 0 \\
\hline
\end{tabular}


disease process. For example, in AKI, creatinine does not elevate until the injury is well established (14). In this study, serum creatinine as a marker of renal function was used. In a cross-sectional study from Japan the prevalence of proteinuria (dipstick test $>30 \mathrm{mg} / \mathrm{dL}$ ) and hematuria (dipstick) in general population was $5.3 \%$ and $9.0 \%$ respectively (15). Japanese investigators have reported a change from glomerulonephritis to diabetic nephropathy and hypertensive nephrosclerosis was the most common causes of CKD $(14,16)$. We believe such a deviation has happened in our population too. Some studies did not recommend the community based CKD screening in asymptomatic adults (17), and rather considered it for diabetics or hypertensive population, or those with a family history of diabetes, hypertension, or CKD $(6,18)$.

\section{Conclusion}

The prevalence of kidney disease in our report was in concordance with other national and international results and shows the influence of known CKD risk factors in our society. Screening for kidney disease and education of health givers is very important. Implementation of simple preventive measurements and the early diagnosis are the key elements to combat this rising threat.

\section{Limitations of the study}

The most important limitation of the study was its small sample size due to the pilot study of cohort. We suggest larger studies on this topic.

\section{Authors' contribution}

Study concept, design, and supervision: MRA and MS. Acquisition of data: FM. Analysis and interpretation of data: EF and FM. Drafting of the manuscript: MRA, EF, and FM. Critical revision of the manuscript for important intellectual content: MRA, SZV. Statistical analysis: FM and EF. Administrative, technical and material support by MS and MGN.

\section{Acknowledgements}

The authors are grateful for the financial support of Liver and Gastrointestinal Disease Research Center-Tabriz University of Medical Sciences, Tabriz, Iran, and also we are deeply indebted to all individuals who participated in this study. We appreciate the contribution of all investigators and staffs of Azar Cohort. We deliver our great thank all health workers of Shabestar health center, East-Azarbaijan-Iran for their close collaboration with our project. Also, we would like to thank the Persian Cohort study staff for their technical support.

\section{Conflicts of interest}

The authors declared no potential conflicts of interest with respect to the research, authorship, and/or publication of this article.
Ethical considerations

Ethical issues (including plagiarism, data fabrication, double publication) have been completely observed by the authors.

\section{Funding/Support}

This study was funded by Liver and Gastrointestinal Disease Research Center-Tabriz University of Medical Sciences, Tabriz, Iran (Grant \# 93/48).

\section{References}

1. Andrassy KM. Comments on 'KDIGO 2012 clinical practice guideline for the evaluation and management of chronic kidney disease. Kidney Int. 2013;84:622-3. doi: 10.1038/ki.2013.243.

2. Meguid El Nahas A, Bello AK. Chronic kidney disease: the global challenge. Lancet. 2005; 365:331-40. doi: 10.1016/ S0140-6736(05)17789-7.

3. Liyanage T, Ninomiya T, Jha V, Neal B, Patrice HM, Okpechi I, et al. Worldwide access to treatment for end-stage kidney disease: a systematic review. Lancet. 2015;385:1975-82. doi: 10.1016/S0140-6736(14)61601-9.

4. de Jong PE, Curhan GC. Screening, monitoring, and treatment of albuminuria: Public health perspectives. J Am Soc Nephrol. 2006;17:2120-6. doi: 10.1681/ ASN.2006010097.

5. Weiner DE, Tighiouart H, Amin MG, Stark PC, MacLeod B, Griffith JL, et al. Chronic kidney disease as a risk factor for cardiovascular disease and all-cause mortality: a pooled analysis of community-based studies. J Am Soc Nephrol. 2004; 15:1307-15.

6. Vassalotti JA, Li S, Chen S-C, Collins AJ. Screening populations at increased risk of CKD: the Kidney Early Evaluation Program (KEEP) and the public health problem. Am J Kidney Dis. 2009;53:107-14. doi: 10.1053/j. ajkd.2008.07.049.

7. National KF. K/DOQI clinical practice guidelines for chronic kidney disease: evaluation, classification, and stratification. Am J Kidney Dis. 2002;39:S1-266.

8. Levey AS, Bosch JP, Lewis JB, Greene T, Rogers N, Roth D. A more accurate method to estimate glomerular filtration rate from serum creatinine: a new prediction equation. Ann Intern Med. 1999;130:461-70.

9. Najafi I, Attari F, Islami F, Shakeri R, Malekzadeh F, Salahi R, et al. Renal function and risk factors of moderate to severe chronic kidney disease in Golestan Province, northeast of Iran. PLoS One. 2010;5:e14216. doi: 10.1371/journal. pone.0014216.

10. Cepoi V, Onofriescu M, Segall L, Covic A. The prevalence of chronic kidney disease in the general population in Romania: a study on 60,000 persons. Int Urol Nephrol. 2012;44:213-20. doi: 10.1007/s11255-011-9923-z.

11. Gambaro G, Yabarek T, Graziani MS, Gemelli A, Abaterusso C, Frigo AC, et al. Prevalence of CKD in northeastern Italy: results of the INCIPE study and comparison with NHANES. Clin J Am Soc Nephrol. 2010;5:1946-53. doi: 10.2215/CJN.02400310.

12. De Zeeuw D, Hillege HL, De Jong PE. The kidney, a cardiovascular risk marker, and a new target for therapy. Kidney Int Suppl. 2005;S25-9. doi: 10.1111/j.15231755.2005.09805.x. 
13. Otero A, De Francisco A, Gayoso P, García F. Prevalence of chronic renal disease in Spain: results of the EPIRCE study. Nefrologia. 2010;30:78-86. doi: 10.3265/Nefrologia. pre2009.Dic.5732.

14. Wakai K, Nakai S, Kikuchi K, Iseki K, Miwa N, Masakane $\mathrm{I}$, et al. Trends in incidence of end-stage renal disease in Japan, 1983-2000: age-adjusted and age-specific rates by gender and cause. Nephrol Dial Transplant. 2004;19:204452. doi: $10.1093 / \mathrm{ndt} / \mathrm{gfh} 317$.

15. Iseki K, Ikemiya Y, Iseki C, Takishita S. Proteinuria and the risk of developing end-stage renal disease. Kidney Int. 2003;63:1468-74. doi: 10.1046/j.1523-1755.2003.00868.x.
16. Imai $E$, Yamagata $K$, Iseki $K$, Iso $H$, Horio $M$, Mkino $H$, et al. Kidney disease screening program in Japan: history, outcome, and perspectives. Clin J Am Soc Nephrol. 2007;2:1360-6. doi: 10.2215/CJN.00980207.

17. Moyer VA. Screening for chronic kidney disease: US Preventive Services Task Force recommendation statement. Ann Intern Med. 2012;157:567-70. doi: 10.7326/00034819-157-8-201210160-00533.

18. Brown WW, Peters RM, Ohmit SE, Keane WF, Collins A, Chen SC, et al. Early detection of kidney disease in community settings: the Kidney Early Evaluation Program (KEEP). Am J Kidney Dis. 2003;42:22-35.

Copyright $\odot 2018$ The Author(s); Published by Nickan Research Institute. This is an open-access article distributed under the terms of the Creative Commons Attribution License (http://creativecommons.org/licenses/by/4.0), which permits unrestricted use, distribution, and reproduction in any medium, provided the original work is properly cited. 